\title{
A theoretical verification of the integral fluctuation theorem for accelerated colloidal systems in the long-time limit
}

\author{
Yash Lokare $^{1}$ \\ Indian Institute of Technology, Delhi, New Delhi, 100016, India
}

\begin{abstract}
A quantitative description of the violation of the second law of thermodynamics in relatively small classical systems and over short time scales comes from the fluctuation-dissipation theorem. It has been well established both theoretically and experimentally, the validity of the fluctuation theorem to small scale systems that are disturbed from their initial equilibrium states. Some experimental studies in the past have also explored the validity of the fluctuation theorem to nonequilibrium steady states at long time scales in the asymptotic limit [see [1]]. To this end, a theoretical and/or purely numerical model of the integral fluctuation theorem has been presented. An approximate general expression for the dissipation function has been derived for accelerated colloidal systems trapped/confined in power-law traps. Thereafter, a colloidal particle trapped in a harmonic potential (generated by an accelerating one-dimensional optical trap) and undergoing Brownian motion has been considered for the numerical study. A toy model of a quartic potential trap in addition to the harmonic trap has also been considered for the numerical study. The results presented herein show that the integral fluctuation theorem applies not only to equilibrium steady state distributions but also to nonequilibrium steady state distributions of colloidal systems in accelerated frames of reference (as opposed to the experimental analysis of said theorem and/or its implications in an inertial frame, as presented in [1]) over long time scales.
\end{abstract}

Keywords: Time-reversed trajectory, colloidal system, integral fluctuation theorem, Langevin equation, dissipation function, power-law traps

\section{Introduction}

The behavior of classical systems under the influence of external perturbations and microscopic forces (both, directed and unavoidable) has been a subject of longstanding interest amongst both, physicists and chemists alike. They seek to quantify the dynamics of such systems by accounting

${ }^{1}$ Email address: ph1180857@iitd.ac.in (Yash Lokare) for the effects of all external perturbations and ascertain if a closed system of equations of motions can be derived that convincingly describe the behavior of the system, at least up to a reasonable approximation. Newton's equations of motion for instance, are time reversible, by which it is implied that for 
every classical trajectory, it is possible to construct a time-reversed trajectory that also serves as a solution to the classical equations of motion [1]. In other words, these timereversed trajectories signify the theoretical reversibility of a system. This implies that the higher the probability of observing timereversed trajectories in a classical system, the greater will be its 'reversibility'. In this specific context, the standard form of the second law of thermodynamics states that a thermodynamic system always evolves in a time-forward direction in an irreversible manner, i.e., no time-reversed trajectories can be observed in a system. It must be noted however, that the second law of thermodynamics in its most general sense applies solely to large, macroscopic systems over time scales that are much longer as compared to those that we consider in the micro- and the nano-scale length limits. We thus arrive at a very fundamental question that can be stated as: How irreversible are thermodynamic systems of interest at such short length scales? The degree of irreversibility of systems at short length scales can enable them to be considered as promising candidates for the realization of novel nanomachines and biological motors that can essentially be reverse engineered in some sense, i.e., the requirement of external power sources to run such small-scale machines is eliminated, unlike conventional large-scale machines that rely on external power sources for their operation. Very early works of Loschmidt et al. and coworkers focused on deriving irreversible macroscopic equations of motion from reversible equations of motion that are relevant at the microscopic scale. Evans et $a l$. pioneered early works on understanding and quantifying the fluctuation theorem [see [2]] which provides a quantitative description of the temporal evolution of a system's irreversibility from a timereversible system at relatively short time scales to a time-irreversible system (in the thermodynamic limit) at very long time scales. In a more general sense, the fluctuation theorem relates the probabilities of observing conjugate time-reversed trajectories and their corresponding 'timeforward' trajectories of a specific time span, say $t$ which are each characterized by a socalled dissipation function (we denote the dissipation function as $\sigma_{t}$ ). The dissipation function is such that it takes on positive and negative values both, for which the following condition holds for some fixed value $\alpha[1]$

$$
P\left(\sigma_{t}=-\alpha\right) / P\left(\sigma_{t}=\alpha\right)=\exp (-\alpha) .
$$

In general, we note that the dissipation function $\sigma_{t}$ assumes a different form for different systems. Eq. (1) clearly demonstrates that with the increasing passage of time (or an upscaling in the size of the system, important due in part to the fact that $\sigma_{t}$ is extensive), the probability of observing time-reversed trajectories that supposedly violate the second law of thermodynamics becomes increasingly rare and this probability thus dies down to nearzero after a sufficiently long time. Also, given the positivity of the RHS of Eq. (1), we note that the ensemble average of $\sigma_{t}$ is always positive and this holds regardless of the size of the system under consideration [1]. The time scales over which the complete dynamics of the irreversibility of a system can be captured is however not determinable from the fluctuation theorem.

In this paper, we focus our attention to the application of the steady-state fluctuation theorem to a numerical model of a colloidal 
system that accelerates along one-

dimension. The reader must note that a separate form of the fluctuation theorem, namely the transient fluctuation theorem (or TFT for short) exists in literature as well.

The transient fluctuation theorem is primarily applicable to those systems that evolve from an initial equilibrium state to a nonequilibrium steady state. The steadystate fluctuation theorem, on the other hand, applies to systems in which one considers the numerical and/or experimental estimation of the dissipation function over certain transient classical trajectories that are evaluated over fixed durations, say $t$ (here too, one starts from an initial equilibrium state). These transient trajectories are sampled out of a parent trajectory under nonequilibrium steady-state conditions (such conditions being maintained over all times). An evaluation over steady-state transient trajectories tells us that the fluctuation theorem holds true only in the long-time asymptotic limit, such that the limiting value of $P\left(\sigma_{t}=-\alpha\right) / P\left(\sigma_{t}=\alpha\right)$, i.e., in the limit $t \rightarrow \infty$ approaches the RHS of Eq. (1) [1]. It is possible to see that certain simplifying approximations made in the argument of $\sigma_{t}$ lead to the asymptotic long-time limit in the steady-state fluctuation theorem. An exact form of the dissipation function can be determined as well [see [1] for details], although in this paper, we solely focus on the derivation and subsequent application of an approximate form of the dissipation function for the colloidal system under consideration. The system that is being considered for the present analysis is such that it would be possible to determine an approximate form for the dissipation function within the realm of deterministic classical dynamics, although an exact, closed-form expression for the dissipation function can be arrived at when one incorporates the framework of Langevin dynamics into the problem at hand (note: we do not concern ourselves with the derivation and analysis of the exact form of the dissipation function in our numerical analysis). The numerical model is based on the drag experiment by Wang et al. [see [3]] in which a colloidal particle was confined/trapped in a harmonic potential generated by a linearly translating optical trap moving at a constant velocity. The numerical analysis presented herein includes an added complexity to the original experiment, i.e., a trap accelerating in the observer's frame of reference has been considered, starting from an initial equilibrium state at time $t=0$. Initially, the colloidal particle is allowed to equilibriate in the optical trap, as a consequence of which its position in the trap can be described by an equilibrium Boltzmann distribution, with the average velocity being zero. The trap (and in turn, the colloidal particle) are then disturbed from their initial equilibrium state by setting them in motion along one dimension. After some time, a situation arises wherein the average acceleration of the particle inside the optical trap becomes nearly equal to the trap's acceleration, thus establishing a nonequilibrium steady-state for the system under consideration. The particle's position (on an average) can be determined by balancing the optical force due to the trap and the hydrodynamic force that the colloidal particle experiences due to its movement through the viscous medium. The observer is placed in the frame of the moving colloidal particle, such that the need of including effects arising from pseudoforces can be eliminated. Here, through a numerical analysis, we demonstrate the 
validity of the fluctuation theorem in the long-time limit (in an accelerating frame of reference) for the case when an approximate form of the dissipation function is considered.

Along the same lines, we then consider the case of a quartic potential trap and demonstrate that the fluctuation theorem holds in the long-time limit when an approximate form of the dissipation function is considered.

\section{A short note on the Langevin equation}

For visualizing Brownian motion, we start by implementing a stochastic component to the force acting on the Brownian particle, a description of which is known only probabilistically. For the velocity of the particle, herein denoted as $v(t)$, we have the following first-order differential equation

$$
m v^{\prime}(t)+\gamma v(t)=f(t),
$$

where $m$ is the mass of the Brownian particle and the prime denotes the first-order derivative of the velocity $v$ with respect to time. Note that in Eq. (2), $f(t)$ denotes the stochastic component of the force acting on the Brownian particle, for which we have $\langle f(t)\rangle=0$. Here, $\gamma v(t)$ denotes the systematic component of the total force acting on the particle. Eq. (2) represents a Langevin equation for the velocity $v$ of the Brownian particle. We assume that the stochastic component of the force $f(t)$ is uncorrelated with the particle velocity $v\left(t_{0}\right)$ for time $t>t_{0}$, i.e., we have $\left\langle f(t) v\left(t_{0}\right)\right\rangle=$ 0 for time $t>t_{0}$. We consider the dynamics of the Brownian particle only on time scales on the order of $m / \gamma$ during which the
Brownian particle suffers a significant number of molecular collisions/interactions. In this context then, we can assume that the stochastic component of the force $f(t)$ behaves essentially as a Gaussian white noise term.

A general solution to the Langevin equation is hard to come by, due to the highly nontrivial nature of $f(t)$. In any case however, we are primarily concerned with meansquare values and certain low order correlation functions. We recast Eq. (2) into the form

$$
v^{\prime}(t)+v(t) / t_{s}=\beta(t),
$$

where we define $\beta(t)$ as $\beta(t) \equiv f(t) / m$ and $t_{s}$ as $t_{s} \equiv m / \gamma$. We naively state that the stochastic component of the force acting on the Brownian particle is uncorrelated with the velocity $v$. However, we must note that a non-zero response of the velocity to $f(t)$ does exist, given which we have

$$
\left\langle\beta(t) v\left(t_{0}\right)\right\rangle \neq 0 ; t<t_{0} .
$$

For $t>t_{0}$, the correlation between $f(t)$ and $v(t)$ vanishes. We assume now that we have a particle whose velocity is known precisely at time $t=0$. We thus have

$$
\begin{aligned}
& v(t)=v(0) \exp \left(-t / t_{s}\right)+\exp (-t / \\
& \left.t_{s}\right) \int_{0}^{t} \exp \left(t_{0} / t_{s}\right) \beta\left(t^{\prime}\right) d t^{\prime} .
\end{aligned}
$$

We know that $\langle\beta\rangle=0$. This then gives us the mean of $v(t)$, as

$$
\langle v(t)\rangle=v(0) \exp \left(-t / t_{s}\right) .
$$

For the mean-square velocity of the Brownian particle, we have

$$
\begin{aligned}
& \left\langle v^{2}(t)\right\rangle=v^{2}(0) \exp \left(-2 t / t_{s}\right)+ \\
& \exp \left(-2 t / t_{s}\right) \int_{0}^{t} \int_{0}^{t} \exp \left(\left(t^{\prime}+t^{\prime \prime}\right) / t_{s}\right)\left\langle\beta\left(t^{\prime}\right) \beta\left(t^{\prime \prime}\right)\right\rangle
\end{aligned}
$$


Note that the in Eq. (7), we eliminate the cross term by making use of the fact that $\langle v(0) \beta(t>0)\rangle=0$. Computing the double integral in Eq. (7) by making an appropriate substitution gives us for the mean-square velocity

$\left\langle v^{2}(t)\right\rangle \approx v^{2}(0) \exp \left(-2 t / t_{s}\right)+t_{s}(1-$

$\left.\exp \left(-2 t / t_{s}\right)\right) \int_{0}^{\infty}\left\langle\beta(0) \beta\left(t^{\prime}\right)\right\rangle d t^{\prime}$.

We note that the mean-square velocity must approach $k_{B} T / m$ at very long times (by the equipartition theorem), given which we get for the variance of the mean-square velocity

$$
\left\langle\sigma_{v}^{2}(t)\right\rangle=k_{B} T / m\left(1-\exp \left(-2 t / t_{s}\right)\right) .
$$

We can follow a similar procedure to compute the mean-square displacement of the Brownian particle. For this, we consider

$$
x v=x(d x / d t)=1 / 2\left(d^{2} x / d t^{2}\right),
$$

which on further simplification gives us

$$
x d v / d t=d(x v) / d t-v^{2}=1 / 2\left(d^{2} x^{2} /\right.
$$$$
\left.d t^{2}\right)-v^{2} \text {. }
$$

We note that the stochastic component of the force is uncorrelated with the position of the Brownian particle at all times, i.e., $\left\langle\beta(t) x\left(t^{\prime}\right)\right\rangle=0$, given which we get from Eq. (10.b)

$d^{2}\left(\left\langle x^{2}\right\rangle\right) / d t^{2}+1 / t_{s}\left(d\left(\left\langle x^{2}\right\rangle\right) / d t\right)=$ $2\left\langle v^{2}\right\rangle$

We consider an equilibrium state in which the mean-square velocity of the Brownian particle assumes the value $k_{B} T / m$. Integrating between appropriate limits thus gives us for the mean-square displacement

$\left\langle x^{2}(t)\right\rangle=2 k_{B} T t_{s}^{2} / m\left[t / t_{s}-(1-\right.$ $\left.\left.\exp \left(-t / t_{s}\right)\right)\right]$.

The stochastic dynamics of a Brownian particle under equilibrium steady-state conditions can hence be analyzed up to a very good approximation.

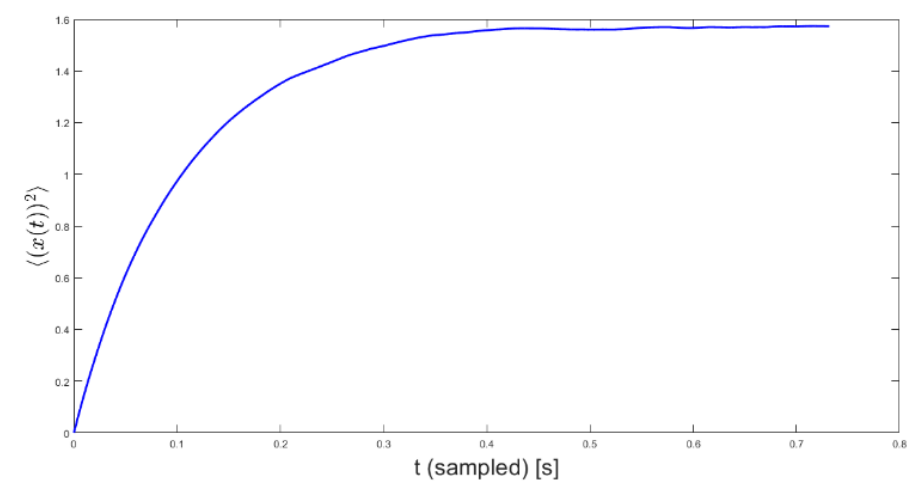

Fig 1. (Color outline) Plot for the mean-square displacement of a colloidal particle confined in a quartic potential well. The colloidal particle undergoes Brownian motion in the trap, while being immersed in a viscous liquid medium. We consider a viscous medium with friction constant $\gamma=0.1$, a trap constant $k=0.967$ and a value of $k_{B} T$ set as $k_{B} T=0.772$ (arbitrary units). The Einstein diffusion constant is given as $D=k_{B} T / \gamma$. A time step of $10^{-3}$ has been considered. We see that this plot conforms well with Eq. (11.b), i.e., the mean-square displacement assumes an asymptotic value after a sufficiently long time span.

\section{Derivation of an approximate form of the dissipation function for steady-state transient trajectories and arbitrary power-law trapping potentials}

For a classical Newtonian system, it is customary to describe the system's state by an arbitrary point in phase space, which consists of the coordinates $\boldsymbol{q}$ and momenta $\boldsymbol{p}$ of all molecules and/or particles that comprise the system. A point in phase space is generally represented as $\boldsymbol{\rho} \equiv\{\boldsymbol{q}, \boldsymbol{p}\}$. The Newtonian equations of motion are timereversible, which implies that for every classical trajectory that exists between points $\boldsymbol{\rho}_{0} \equiv\left\{\boldsymbol{q}_{0}, \boldsymbol{p}_{0}\right\}$ and $\boldsymbol{\rho}_{t^{*}} \equiv\left\{\boldsymbol{q}_{t^{*}}, \boldsymbol{p}_{t^{*}}\right\}$, there exists a time-reversed trajectory between points $\boldsymbol{\rho}_{0}^{\prime} \equiv\left\{\boldsymbol{q}_{t^{*}},-\boldsymbol{p}_{t^{*}}\right\}$ and $\boldsymbol{\rho}_{t^{*}}^{\prime} \equiv$ $\left\{\boldsymbol{q}_{0},-\boldsymbol{p}_{0}\right\}$. We now define a volume element for a set of trajectories at some arbitrary 
time $t^{\prime}$, as $d V\left(\boldsymbol{\rho}_{t^{\prime}} \equiv\left\{\boldsymbol{q}_{t^{\prime}}, \boldsymbol{p}_{t^{\prime}}\right\}\right)$, corresponding to which we will have a volume element for a set of time-reversed trajectories, which we denote as $d V\left(\boldsymbol{\rho}_{t^{\prime}}^{\prime} \equiv\right.$ $\left.\left\{\boldsymbol{q}_{t-t^{\prime}},-\boldsymbol{p}_{t-t^{\prime}}\right\}\right)$ [1]. As discussed in section I [see Eq. (1)], the reversibility of a system can be defined as the ratio of the probabilities of observing time-reversed trajectories and their corresponding timeforward trajectories. It is however a daunting task to deduce closed-form expressions for arbitrary nonequilibrium steady-state distributions in phase space [1]. We instead evolve the system (and in turn, the transient trajectories) from an equilibrium steady-state distribution, for which the dynamics is well known, i.e., we start the temporal evolution of the system at time $t^{\prime}=0$. The dissipation function $\sigma_{t}(\rho)$ thus assumes the form [1]

$\sigma_{t}(\rho)=\ln \left(P\left[d V\left(\rho_{0}\right)\right] / P\left[d V\left(\rho_{0}^{\prime}\right)\right]\right)$.

We now consider the case of a colloidal system localized in an arbitrary power-law trap which is translating at a constant acceleration in one dimension. At a particular instant $t^{\prime}$, we denote the coordinate and momenta of the colloidal particle as $x_{t^{\prime}}^{*}$ and $p_{t^{\prime}}$ (we consider the motion along the $x$-axis), respectively. This particle is in a viscous medium and the arrangement is such that the force due to the power-law trap does not affect the neighboring solvent molecules and they all remain stationary relative to the moving colloidal particle. Thus, a simplified version of a colloid is being considered in this context. We consider constant temperature conditions, given which it is possible to apply the canonical ensemble to this system. The temperature (constant) is denoted as $T$. The Hamiltonian of the system can be expressed as a sum of the kinetic and potential energies of the system that arise due to intermolecular interactions and the potential energy of the colloidal particle that is trapped/confined in the moving trap. We consider very small displacements of the colloidal particle from its mean equilibrium position (i.e., the focal point of the trap) such that it always remains bound to this potential well. The Hamiltonian of the system for the time-forward trajectories assumes the form

$H\left(\rho_{0}\right)=E_{K}\left(x_{0}\right)+U_{\text {int }}\left(x_{0}\right)+U_{\text {trap }}\left(x_{0}^{*}\right)$,

where $E_{K}\left(x_{0}\right)$ denotes the colloidal system's kinetic energy and $U_{\text {int }}\left(x_{0}\right)$ denotes the system's potential energy arising due to intermolecular interactions, respectively. Here, $U_{\text {trap }}\left(x_{0}^{*}\right)$ denotes the potential energy of the power-law trap in the equilibrium steady-state. We note that $E_{K}\left(x_{0}\right)$ and $U_{\text {int }}\left(x_{0}\right)$ do not undergo any temporal evolution and are thus considered to be constant quantities. The potential of the trap in question assumes the form

$U_{\text {trap }}(x)=k / 2 n\left(x_{r}\right)^{2 n}$,

where $k$ denotes the trap constant and $x_{r}$ denotes the position of the colloidal particle relative to the position of the trap's center. Here, $n$ is an integer. Note that we can obtain bound solutions only for even values of the exponent, i.e., $2 n$. For all oddexponent traps, we fail to obtain any bound solutions (by which it is implied infinite values of the mean-square displacement and so forth) and the colloidal particle simply escapes the influence of the confining potential well. Clearly, we note that $n=1$ corresponds to the case of a harmonic trap and $n=2$ corresponds to the case of a 
quartic potential well. Since the trap is accelerating uniformly, we note that the temporal evolution of the position of the trap center for some constant acceleration $a_{\text {trap }}$ assumes the form

$x_{\text {trap }}=(1 / 2) a_{\text {trap }}\left(t^{*}\right)^{2}$,

where we note that the motion of the trap starts from rest. Given Eq. (15), we have for $x_{r}$

$x_{r}=x-(1 / 2) a_{\text {trap }}\left(t^{*}\right)^{2}$.

Thus, from Eq. (14) and Eq. (16), the complete form of the trap potential can be taken as

$U_{\text {trap }}(x)=k / 2 n\left(x-(1 / 2) a_{\text {trap }}\left(t^{*}\right)^{2}\right)^{2 n}$.

Similarly, for the time-reversed trajectories, the Hamiltonian of the system assumes the form

$H\left(\rho_{0}^{\prime}\right)=E_{K}\left(-p_{t}\right)+U_{\text {int }}\left(x_{t}\right)+U_{\text {trap }}\left(x_{t}^{*}\right)$,

where we have considered a time-reversal in the phase space coordinates. We also take note of the fact that to preserve time-reversal symmetry, we must consider the trap center to be located at the point $(1 / 2) a_{\text {trap }}\left(t^{*}\right)^{2}$ at the start of the temporal evolution of a timereversed trajectory. We note that in a canonical ensemble, the following proportionality relation is satisfied

$P\left[d V\left(\rho_{0}\right)\right] \propto \exp \left(-H\left(\rho_{0}\right) / k_{B} T\right)$.

From Eq. (12) and Eq. (19), we have

$$
\begin{aligned}
& \sigma_{t}(\rho)=\ln \left(\left[\exp \left(-H\left(\rho_{0}\right) / k_{B} T\right]\right) /\right. \\
& \left.\left[\exp \left(-H\left(\rho_{0}^{*}\right) / k_{B} T\right]\right)\right),
\end{aligned}
$$

which on further simplification yields $\sigma_{t}(\rho)=1 / k_{B} T\left[H\left(\rho_{t}\right)-H\left(\rho_{0}\right)\right]$,

where we have used the time-reversal symmetry of time-forward and timereversed transient trajectories. We can further simplify Eq. (20.b) by converting it into an integral form, as follows

$\sigma_{t}(\rho)=1 / k_{B} T \int_{0}^{t}\left(\frac{d H\left(\rho_{t^{*}}\right)}{d t^{*}}\right) d t^{*}$.

The term in the parenthesis of the integrand in Eq. (20.c) can be expressed as the sum of the differentials of the three energy terms defined in the Hamiltonian of the system [see Eq. (13) and Eq. (18)]. We consider these individually as follows

$$
\begin{aligned}
& \int_{0}^{t} \frac{d U_{\text {trap }}\left(x_{r}\right)}{d t^{*}} d t^{*}=\int_{0}^{t} k(x- \\
& \left.\left(\frac{1}{2}\right) a_{\text {trap }}\left(t^{*}\right)^{2}\right)^{2 n-1}\left(\frac{d x}{d t^{*}}-a_{\text {trap }} t^{*}\right) d t^{*},
\end{aligned}
$$

and a similar expression (with the sign of one of the terms reversed) can be found for the kinetic term. After much simplification, we arrive at the following result for the dissipation function $\sigma_{t}(\rho)$, as

$$
\begin{aligned}
& \sigma_{t}(\rho)=a_{\text {trap }} k / k_{B} T \int_{0}^{t}(x- \\
& \left.\left(\frac{1}{2}\right) a_{\text {trap }}\left(t^{*}\right)^{2}\right)^{2 n-1} t^{*} d t^{*} .
\end{aligned}
$$

Here, we note that $x$ denotes the position of the colloidal particle inside the trap at intermediate instances of time. The result in Eq. (20.e) is a general result (although approximate) that holds for all power-law traps. In the present analysis, we consider cases of the harmonic trap and the quartic potential trap, for which we have $n=1$ and $n=2$, respectively. Note that the acceleration of the trap, $a_{\text {trap }}$ is constant 
throughout, and we place the observer in the center-of-mass frame of the moving colloidal particle/trap, given which the contributions of pseudo-force terms can be eliminated. We use Eq. (20.e) for the numerical analysis of the integral fluctuation theorem for such a system.

\section{Numerical results}

\section{A. Harmonic trap:}

For this, we allow the system to reach a steady-state equilibrium distribution initially. This results in a MaxwellianBoltzmann distribution for the particle velocities inside the trap before the trap starts accelerating. The optical trap thus generated has a stiffness constant of $k=$ $3.87 \times 10^{-7} \mathrm{~N} / \mathrm{m}$. We consider the viscosity of the medium to be $\eta=2.5$ Poise and the radius of the colloidal particle to be $R=$ $9.23 \mu \mathrm{m}$. The temperature of the system is maintained at $433 \mathrm{~K}$. The friction constant for the medium is given as $\gamma=6 \pi \eta R$. The stochastic component of the force has been modeled by a Gaussian random number generator in the simulation. The whole system is set in motion for about 20 seconds starting from $t=0$, from which we sample out 5000 transient particle trajectories, each spaced at a time interval of $4 \times 10^{-3}$ seconds, i.e., a time step of $\Delta t=4 \times 10^{-3}$ seconds has been implemented in the numerical analysis. We consider a constant acceleration of $a_{\text {trap }}=4 \times 10^{-7} \mathrm{~m} / \mathrm{s}^{2}$ for the optical trap under consideration. The setup is such that the initial position of the colloidal particle is at a distance of $0.85 \mu \mathrm{m}$ from the bottom of the sample cell, whereas we take the initial distance of the trap center from the bottom of the sample cell to be
$0.35 \mu \mathrm{m}$. We make use of Eq. (2) to compute the transient trajectories of the colloidal particle over a time interval of 20 seconds (and in turn, the positions of the optical trap center relative to the colloidal particle and the walls of the sample cell at intermediate time instances). A histogram plot for the stochastic entropies generated and lost along transient trajectories is plotted. A loss of entropy essentially implies the reduction of the kinetic energy of the colloidal particle inside the trap over an intermediate time step, which is lost to the viscous medium. These are what constitute the set of timereversed transient trajectories. Eq. (20.e) has been used to compute the stochastic entropies along transient trajectories (by substituting $n=1$ ).

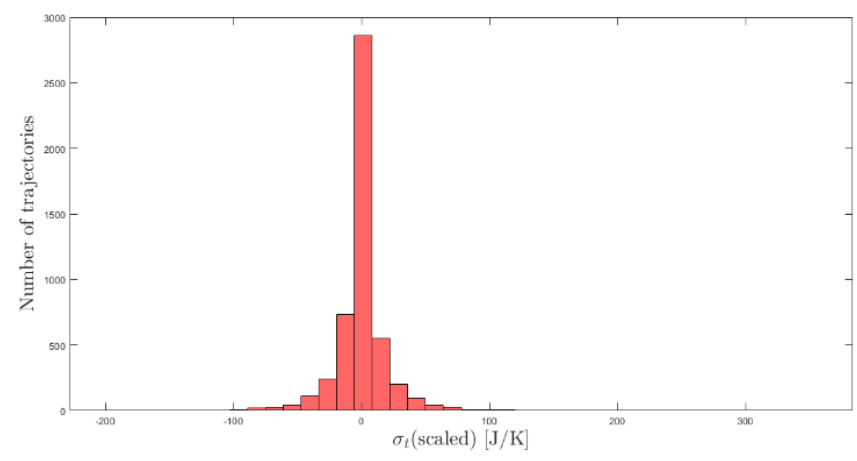

Fig 2. (Color outline) Histogram plot for the stochastic entropy distribution of the colloidal system along transient Brownian trajectories (plotted against scaled values of $\sigma_{t}$ ). We see that the stochastic entropy distribution assumes a Gaussian profile and conforms well with all theoretical predictions [see Eq. (3) in [4]] The parameters considered for generating the plot have been quoted in the main text.

To check the validity of the integral fluctuation theorem, which can be expressed mathematically as [3]

$P\left(\sigma_{t}<0\right) / P\left(\sigma_{t}>0\right)=\exp \left(-\sigma_{t}\right)_{\sigma_{t}>0}$,

we sample out the positive values of the stochastic entropy (i.e., entropy generation) and plot these results against time $t$. 


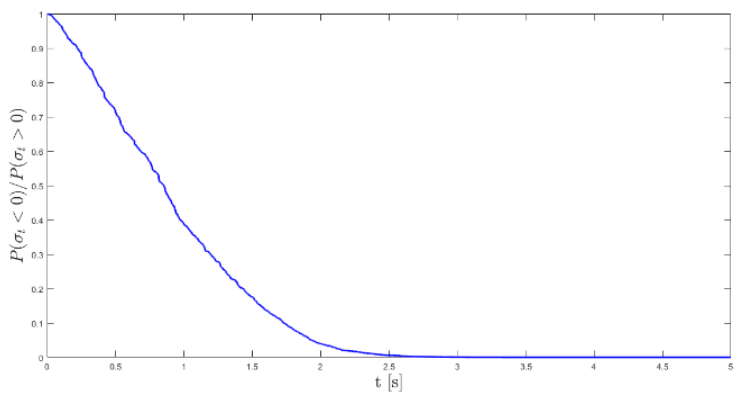

Fig 3. (Color outline) Plot for the number ratio (i.e., ratio of the probability of observing time-reversed trajectories to the probability of observing time-forward trajectories) versus time. We see that the RHS of Eq. (21) holds true in the simulation results, thus demonstrating that the integral fluctuation theorem holds for small-scale systems over relatively long-time scales. We observe that beyond 2.5 seconds, the probability of observing time-reversed trajectories practically becomes zero.

\section{B. Quartic potential well:}

We consider a similar scenario in this case. No changes in the parameters have been made and we follow the exact same procedure as we did for the case of the harmonic trap, i.e., Eq. (2) has been used to numerically compute the transient Brownian trajectories over a time interval of about 20 seconds (and in turn, the positions of the optical trap center relative to the colloidal particle and the walls of the sample cell at intermediate time instances). We use Eq. (20.e) to solve for the stochastic entropies generated and/or lost along transient particle trajectories by making the substitution $n=$ 2.

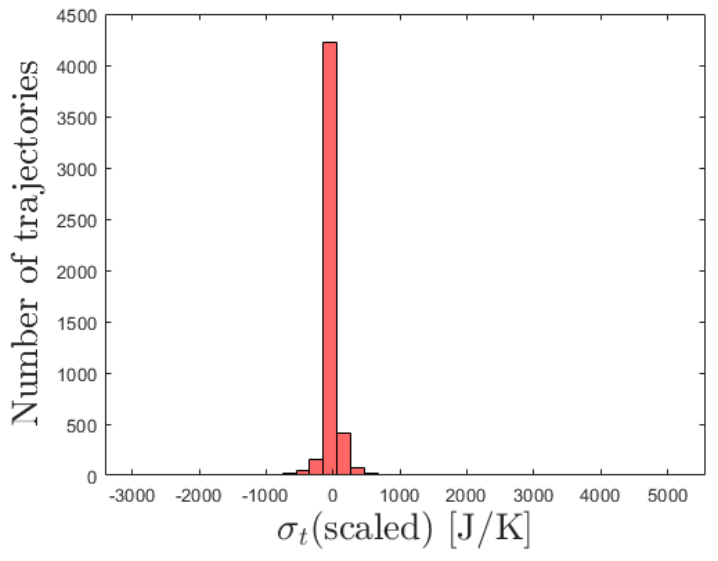

Fig 4. (Color outline) Histogram plot for the stochastic entropy distribution of the colloidal system along transient Brownian trajectories (plotted against scaled values of $\sigma_{t}$ ). Here again, we see that the stochastic entropy distribution assumes a Gaussian profile and conforms well with all theoretical predictions [see Eq. (3) in [4]]. The parameters considered for generating the plot have been quoted in the main text.

As in the case of the harmonic trap, we make use of Eq. (21) to visualize and check the validity of the integral fluctuation theorem. Positive values of $\sigma_{t}$ are sampled out and the number ratio is plotted against time $t$.

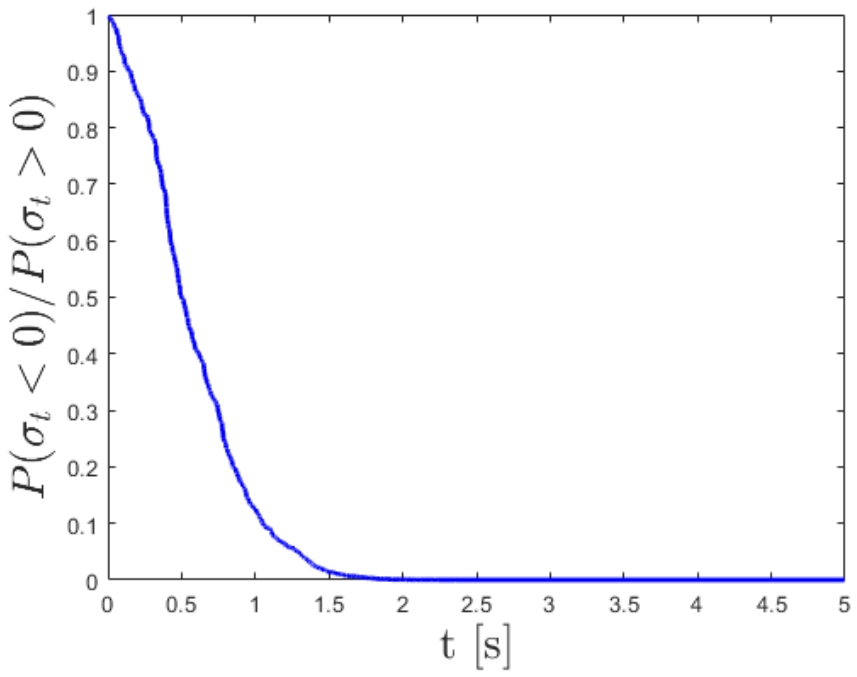

Fig 5. (Color outline) Plot for the number ratio (i.e., ratio of the probability of observing time-reversed trajectories to the probability of observing time-forward trajectories) versus time. We see that the RHS of Eq. (21) holds true in the simulation results, thus demonstrating that the integral fluctuation theorem holds for small-scale systems over relatively long-time scales. We observe that beyond 2 seconds, the probability of observing time-reversed trajectories practically becomes zero. 


\section{Summary}

A detailed mathematical analysis of the integral fluctuation theorem in the context of an accelerated colloidal system has been presented. An approximate closed-form expression for the dissipation function has been derived in the context of a colloidal system, in which a colloidal particle is bound in a potential well and is made to accelerate uniformly along one dimension, while undergoing Brownian motion inside the trap. We note that expression [see Eq. (20.e)] holds true for any power-law trap, hence can be generalized and analyzed beyond what has been presented in this paper. Two cases, one corresponding to a harmonic trap and the other corresponding to a quartic potential well have been considered and numerically analyzed. The results thus obtained show that the integral fluctuation theorem holds true for the form of the dissipation function deduced herein in the long-time limit. An exact, closed form generic expression for the dissipation function can also be derived using stochastic dynamics [see [1] for details]. We conclude by saying that novel nanomachines and biological motors can indeed be realized in practice and this is no longer a scientific myth, as has been demonstrated in the above results.

\section{Acknowledgements}

The author (Y.L.) wishes to express his gratitude to his mentor, D.R.M Williams for his continued and generous support. This research did not receive any specific grant from funding agencies in the public, commercial or not-for-profit sectors.
Conflict of interest: The author declares no conflict of interest with any third party.

\section{References}

[1] G. M. Wang, J. C. Reid, D. M. Carberry, D. R. M. Williams, E. M. Sevick, and D. J. Evans, "Experimental study of the fluctuation theorem in a nonequilibrium steady state," Phys. Rev. E - Stat. Nonlinear, Soft Matter Phys., vol. 71, no. 4, 2005, doi: 10.1103/PhysRevE.71.046142.

[2] D. J. Evans, E. G. D. Cohen, and G. P. Morriss, "Probability of Second Law Violations in Shearing Steady States," Phys. Rev. Lett., vol. 71, no. 21, pp. 3616-3616, Nov. 1993, doi: 10.1103/PhysRevLett.71.3616.

[3] G. M. Wang, E. M. Sevick, E. Mittag, D. J. Searles, and D. J. Evans, "Experimental Demonstration of Violations of the Second Law of Thermodynamics for Small Systems and Short Time Scales," Phys. Rev. Lett., vol. 89, no. 5, 2002, doi: 10.1103/PhysRevLett.89.050601.

[4] T. Speck, V. Blickle, C. Bechinger, and U. Seifert, "Distribution of entropy production for a colloidal particle in a nonequilibrium steady state," Epl, vol. 79, no. 3, pp. 1-5, 2007, doi: 10.1209/02955075/79/30002. 